\title{
Description of an Unknown Species, Rotylenchus arsenjevi and Additional Information on Geocenamus adakensis (Tylenchida : Nematoda) from Korea
}

\author{
Joong-Hwan Lee*, Seok-Hee Park, So-Deok Park and Tae-Young Kwon \\ Gyeongbuk Agricultural Technology Administration, Dongho-dong, Daegu, 702-708
}

\section{한국 미기록종 Rotylenchus arsenjevi 기재 및 Geocenamus adakensis에 관한 추가 정보 \\ 이중환* · 박석희 · 박소득 · 권태영 \\ 경상북도 농업기술원}

\begin{abstract}
Rotylenchus arsenjevi are reported for the first time in Korea. The Korean species differs slightly by having a hemispherical lip region and a slightly shorter tail than the original description ( $\mathrm{c}=38.2-68.7$ vs 36-41). Male was founded firstly from Korea and described. Geocenamus adakensis were collected a second time from Korea at a different locality and habitat. The measurements were compared with original description and the firstly collected specimen in Korea.
\end{abstract}

Key words: Taxonomy, Geocenamus adakensis, Rotylenchus arsenjevi.

초 록: 나선선충과의 한국 미기록종 Rotylenchus arsenjevi를 기재하였다. 한국 종은 구순부가 반원형이고 미장이 약간 짧 고, 수컷은 처음으로 채집되었다. Geocenamus adakensis는 한국에서 두번째로 채집되었다. 이번에 채집된 종의 측정치를 원 기재 종 및 지난번 채집한 종과 비교하였다.

검색어: 분류, Geocenamus adakensis, Rotylenchus arsenjevi 기재

\section{Materials and Methods}

Soil samples were collected from soil around roots of mulberry tree (Morus bombycis Koidz) at Odae mountain and Allium vitorialis var. platphyllum Makino at Ulreung Island. Nematodes were extracted by modified sieving method. Fixed with $70^{\circ} \mathrm{C}$ F:G 4-1 fixative, then transferred to anhydrous glycerine by Seinhorst's rapid glycerine method(Seinhorst, 1959). Nematodes were mounted using paraffin ring double cover glass method. Measurements and drawings were made using a drawing tube attached to an Olympus BX 50 microscope. Photographs was taken

*Corresponding author: 1jh8888@korea.kr Received December 11 2009; revised March 22 2010; accepted April 142010 using differential interference contrast attachments.

\section{Description}

Rotylenchus arsenjevi (Eroshenko, 1984) Maggenti et al, 1988(울릉나선선충)(신칭)

(Figs. 1, 2)

\section{Measurements:}

Female $(n=5)$ : $L=765 \mu m(695-962) \mu m ; a=30.2(25.7-34.9)$; b'=5.9(5.4-7.3); c=55.1(38.2-68.7); c'=0.7(0.5-1.0); V=59.1 (55.3-66.4); Stylet=27.6 $\mu \mathrm{m}$ (20.5-30.5); Body width $=25.3 \mu \mathrm{m}$ (22.5-28.0); Oesophagus length $=127.3 \mu \mathrm{m}(121.2-131.2)$; Anterior end to excretory pore $=114.9 \mu \mathrm{m}(112.5-117.5)$; DGO= 5.9 $\mu \mathrm{m}(3.5-8.5)$ : Tail length $=14.4 \mu \mathrm{m}(11-19)$.

Male $(n=3)$ : $L=680 \mu m(655-707) ; a=30.7(29.1-33.6) ; b '=5.7$ 


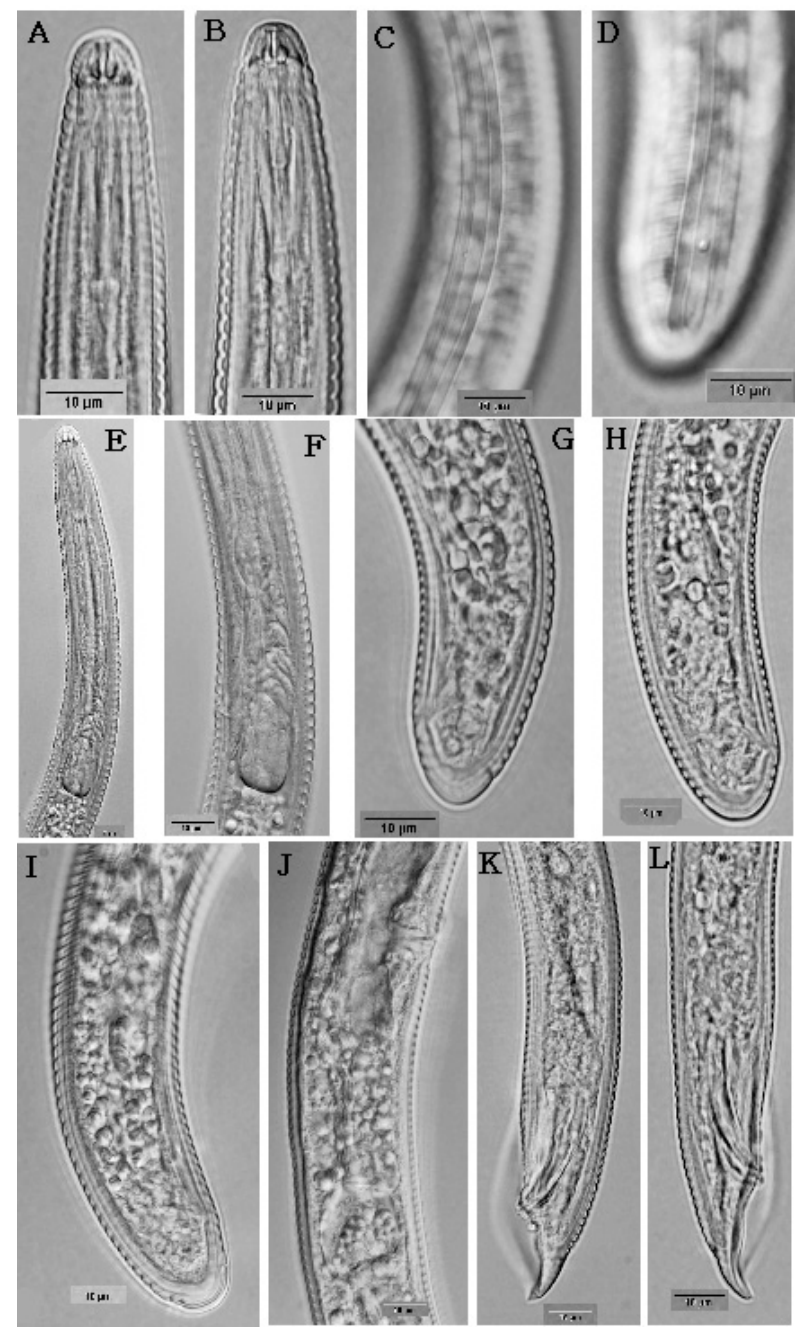

Fig. 1. Rotylenchus arsenjevi: A: Female head; B: Male head; C: Lateral field at middle of body; D: Lateral field at tail, showing phasmid; E: Oesophageal region; $F$ : Enlargement of posterior part of oesophagus; G-I: Female posterior part; J: Posterior female gonad, showing sperm in the spermatheca; $K$ and $L$ : Male posterior part.

(5.6-5.8); $\quad \mathrm{c}=32.6(28.3-36.2) ; \quad \mathrm{c}^{\prime}=1.2(1.1-1.4) ; \quad$ Stylet $=27.7 \mu \mathrm{m}$ (27.5-28.0); $\mathrm{DGO}=5.9 \mu \mathrm{m}(3.5-8.5)$; Anterior end to excretory pore $=101 \mu \mathrm{m}(98.5-103.5)$; Tail length $=21 \mu \mathrm{m}(19.5-24.0)$; Spicule $=$ 27.3 $\mu \mathrm{m}(25-30)$; Gubernaculum $=9.5 \mu \mathrm{m}(9-10)$.

Female: Body spiral when fixed. Labial region setoff, hemispherical with 6 annules. Labial framework strongly sclerotized. Stylet knobs rounded. DGO 3.5-8.5 $\mu \mathrm{m}$ from stylet base. Excretory pore located at 113-118 $\mu \mathrm{m}$ from anterior end. Terminal oesophageal bulb slightly overlap intestine dorsally. Lateral field with 6 incisures, out line areolated at oesophageal region. Female reproductive

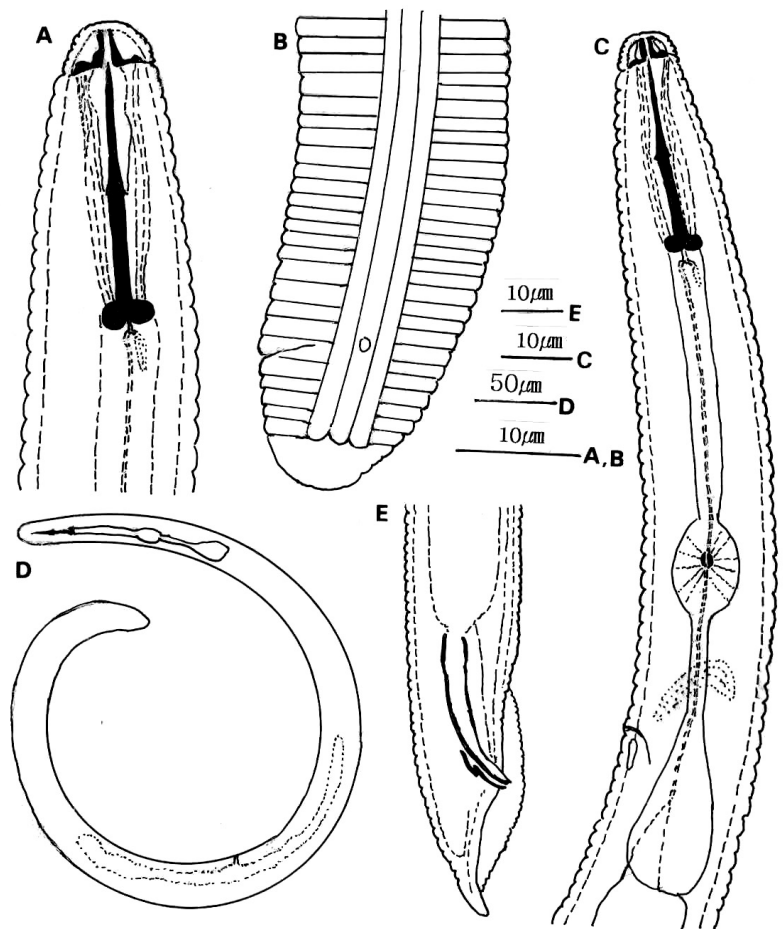

Fig. 2. Rotylenchus arsenjevi: A: Head; B: Surface view of female posterior region; C: Female anterior region; D: Entire female; $E$ : Male posterior region.

system didelphic. Spermatheca rounded without spermatozoa. Vulva lip with epyptigma. Tail short with 4-6 annules in ventral side. Tail terminus smooth rounded or conical. Phasmids at anus level.

Male: Male similar to female in general shape. Body ventrally curved when fixed. Bursa crenate, envelop tail. Spicule 25-30 $\mu \mathrm{m}$ long, gubernaculum 9-10 $\mu \mathrm{m}$ long(Casttillo et al., 1993).

Locality and host plant: Soil around the roots of Allium vitorialis var. platphyllum Makino at Ulreung island.

Discussion: Korean specimen well correspond to the description of $R$. arsenjevi (Eroshenko, 1984) Maggenti et al, 1988 except lower number of annules on tail(4-6 vs 7-9 in $R$. arsenjevi). Male firstly founded in Korea.

Gecenamus adakensis (Bernard, 1984) Brzeski, 1991. (Fig. 3)

Measurements: See table 1, 2.

Geocenamus adakensis (Bernard, 1984) Brzeski, 1991 were found for the second time since Choi \& Geaert reported 


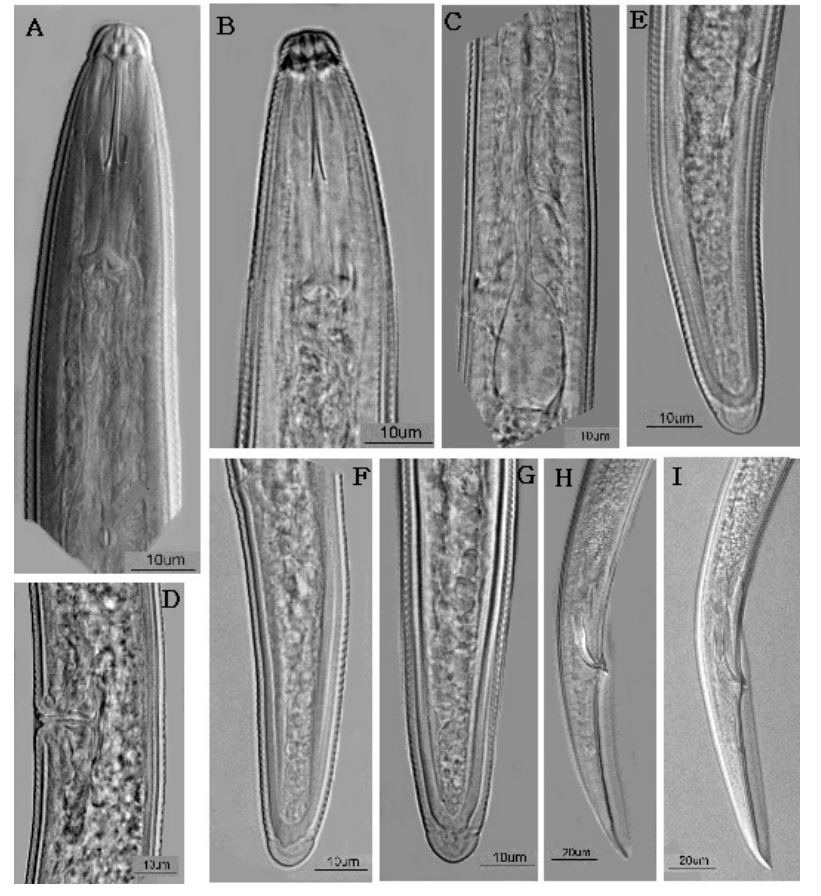

Fig. 3. Geocenamus adakensis: A, B: Head region; C: Oesophageal region; D: Vulva region; E-G: Various shape of female tail; $H$ and $I$ : Male tail.

Table 1. Comparative measurements of female Geocenamus adakensis at different localities

\begin{tabular}{lccc}
\hline \hline & $\begin{array}{c}\text { Paratype* } \\
\mathrm{n}=22\end{array}$ & $\begin{array}{c}\text { Specimens } \\
\mathrm{n}=11\end{array}$ & $\begin{array}{c}\text { Specimens 2 } \\
\mathrm{n}=12\end{array}$ \\
\hline $\mathrm{L}(\mu \mathrm{m})$ & $959-1284$ & $1025-1405$ & $773-915$ \\
Stylet $(\mu \mathrm{m})$ & $32-36$ & $33-37$ & $34.0-39.0$ \\
D.G.O. $(\mu \mathrm{m})$ & $1.4-3.3$ & $2-4$ & $1.4-3.1$ \\
$\mathrm{a}$ & $27-37$ & $27-37$ & $25.1-32.1$ \\
$\mathrm{~b}$ & $5.5-6.8$ & $5.8-7.5$ & $4.9-6.8$ \\
$\mathrm{c}$ & $10.6-14.2$ & $12-16.2$ & $11.1-13.7$ \\
$\mathrm{c}^{\prime}$ & $3.1-4.1$ & $2.6-3.7$ & $2.8-3.7$ \\
$\mathrm{~V}(\%)$ & $51-58$ & $51.2-55.0$ & $51.7-56.0$ \\
$\mathrm{~m}(\%)$ & $50-55$ & $50.0-61.4$ & \\
Tail length $(\mu \mathrm{m})$ & $71-108$ & $64.0-80.5$ & \\
No. of tail ann. & $35-65$ & $49-57$ & \\
Lip annuli & $6-8$ & 6 & $6-7$ \\
\hline
\end{tabular}

*Paratype (Bernard, 1984); Specimens 1: Collected at Bugwi-myeon, Jeollabuk-do(Latitude N 35 , Longitude E $127^{\circ}$ ), Korea (Choi \& Geraert, 1993); Specimens 2: Collected at Mt. Odae Kangweon-do (Latitude N $37^{\circ}$, Longitude E 128) Korea (in 2008).

in 1993 from Korea. Measurements of the populations from the different localities were compared. All the measurements of both sex are correspond to the original description except body length much shorter in population 2; 773-915 $\mu \mathrm{m}$ vs
Table 2. Comparative measurements of male Geocenamus adakensis at different localities

\begin{tabular}{lccc}
\hline \hline & $\begin{array}{c}\text { Paratype } \\
\mathrm{n}=4\end{array}$ & $\begin{array}{c}\text { Specimens } \\
\mathrm{n}=10\end{array}$ & $\begin{array}{c}\text { Specimens } 2 \\
\mathrm{n}=12\end{array}$ \\
\hline $\mathrm{L}(\mu \mathrm{m})$ & $997-1172$ & $1005-1330$ & $738.5-910.0$ \\
Stylet $(\mu \mathrm{m})$ & $33-35$ & $32-34$ & $30.0-37.1$ \\
D.G.O. $(\mu \mathrm{m})$ & $1.6-2.0$ & $2.4-4.5$ & \\
$\mathrm{a}$ & $31.1-41.3$ & $28.7-35.8$ & $27.3-35.4$ \\
$\mathrm{~b}$ & $5.9-7.5$ & $5.2-6.4$ & $4.9-5.7$ \\
$\mathrm{c}$ & $10.0-10.8$ & $10.8-14.7$ & $8.6-11.4$ \\
$\mathrm{c}$ & $4.1-5.1$ & $3.4-4.8$ & $3.3-6.6$ \\
$\mathrm{~T}(\%)$ & $46-61$ & $31-38$ & \\
$\mathrm{~m}(\%)$ & $53-57$ & $49.1-57.3$ & \\
Spicule $(\mu \mathrm{m})$ & $30-35$ & $31-35$ & $23.0-33.6$ \\
Gubernaculum $(\mu \mathrm{m})$ & $7-10$ & $9-12$ & $9.0-12.6$ \\
\hline
\end{tabular}

959-1,284 $\mu \mathrm{m}$. The body length and tail length of the population 2 were shorter than the populaion 1; 773-915 $\mu \mathrm{m}$ vs $1025-1405 \mu \mathrm{m}$ and $(64.0-80.5 \mu \mathrm{m}$. vs $71-108 \mu \mathrm{m}$ respectively.

Locality and habitat: Soil around roots of mulberry tree(Morus bombycis Koidz) at Odae Mountain.

\section{Acknowledgement}

I thank to a Professor emeritus Y. E. Choi for checking specimens

\section{Literature Cited}

Bernard, E.C. 1984. Hoplolaimoidea (Nematoda: Tylenchida) from the Aleutian islands with descriptions of four new species. J. Nematol., 16(2): 194-203.

Casttillo, P., N. Vovlas, A. Gomez-Barcina and F. Lamberti. 1993. The plant parasitic nematode Rotylenchus (A monograph). Supplement of Nematologia Mediterranea. 21: 1-200. Choi, Y.E. and E. Geraert, 1993. Nematodes associated with forest trees in Korea. II. Three new and one described species of Geocenamus with a note on the en face view in the genus. Nematologica. 39(4): 431-449.

Seinhorst, J.w., 1959. A rapid method for the transfer of nematodes from fixative to anhydrous glycerine. Nematologica. 4: 67-69. 